\title{
Automating Blood Flow Simulation Through the Aorta in Patient-specific CT Images
}

\author{
Marija Habijan \\ Faculty of Electrical Engineering, \\ Computer Science and Information \\ Technology Osijek, Croatia \\ marija.habijan@ferit.hr
}

\author{
Irena Galić \\ Faculty of Electrical Engineering, \\ Computer Science and Information \\ Technology Osijek, Croatia \\ irena.galic@ferit.hr
}

\begin{abstract}
Computational fluid dynamics (CFD) modeling of blood flow is significant for obtaining patient-specific hemodynamics information for functional assessment of the cardiovascular system. In this work, we present a framework for fully automatic CFD simulation through the aorta. The proposed framework consists of four main stages: (1) automatic segmentation of the aorta, (2) model generation, (3) mesh creation, and (4) blood flow simulation. In the segmentation part, we utilized a 3D MultiResUnet network for automatic segmentation of organs at risk from the CodaLab SegThor Challenge. After that, we extract ascending and descending aorta and further proceed with model and mesh generation. Finally, we simulate the pressure along the surface of the aorta, the displacement, and the velocity. The entire framework ${ }^{1}$ was implemented in Python with open-sourced dependencies (Pytorch, VTK, SimVascular, SimpleITK), can be executed from the command line, and does not require user intervention, significantly reducing aorta simulation time.
\end{abstract}

\section{Introduction}

The cardiovascular system, composed of the heart and vessels (aorta, arteries, and veins), distributes oxygen and essential nutrients through the blood to all organs in the human body. Different structures of the cardiovascular system can be affected by various pathologies called cardiovascular diseases, which are the third leading cause of death in the world [1]. In arteriosclerosis, the wall of the vessels sometimes develops abnormalities, called lesions. They often cause vessel narrowing due to the buildup of atheromatous plaque and, in severe cases, can result in coronary

${ }^{1}$ https://github.com/mhabijan/blood_flow_ simulation artery disease, peripheral artery disease, or stroke [2, 3, 4]. Early arteriosclerotic lesions develop in branching regions of vessels where blood flow is significantly disrupted due to vessels' complex geometry and blood flow pulsatile nature [5]. A better understanding of arteriosclerotic development is enabled through image-based modeling, and numerical simulations of aortic blood flow [6].

A typical workflow starts with the manual or semi-automatic segmentation of cardiovascular structures from images obtained by computed tomography (CT) or magnetic resonance (MRI). After that, model generation and meshing are performed to create simulation-ready models. The final step, blood flow simulation, relies on applying computational fluid dynamics (CFD) [7] to previously obtained computer models and enables computation of patient-specific blood flow information, capturing hemodynamic features which are not measurable in vivo. These steps are generally performed using separate software tools and often require significant human effort, which is a time-consuming process subjected to unpredictable and unreproducible errors. Therefore, designing and developing automatic methods for generating blood simulations are necessary to unify and simplify this complex task.

In this work, we present an automated framework for generating aorta CFD simulations from CT volumes. Our framework proposes a 3D MultiResUnet method to perform automatic segmentation of the aorta from CT images. We further automate surface processing to generate volumetric computational models and meshes that are suitable for CFD simulations. Finally, we simulate the pressure along the surface of the aorta, the displacement, and the velocity. The remainder of the paper has the following structure. Section 2 briefly describes the most important prior researches and concepts. In Section 3, we give a detailed description of the proposed framework and used methods. Section 
4 includes experiments and obtained results for both segmentation and simulation results. Finally, in Section 5 , we provide a discussion and give a conclusion.

\section{Related Research}

In these subsections, we review some related works. First, we review the prior methods in the segmentation tasks of thoracic organs at risk since we use the same dataset for aorta extraction in the first part of our framework. After that, we present previous approaches for aorta simulation.

\subsection{Prior Segmentation Methods}

Recent developments in deep learning and the broad attainability of high-performing graphical processing units (GPUs) alleviated automated segmentation of different organs and parts of the human body using convolutional neural networks (CNNs). The commonly used approaches include various modifications and improvements of U-Net architecture [8] and its 3D counterpart, 3D U-Net [9]. For example, Chen et al. [10] use original 2D U-Net network, while He et al. [11] use 2D U-Net architecture with residual blocks. Vesal et al. [12] use the dilated residual network, which expands the receptive field in the lowest level of the network. This enables efficient use of both global and local information without increasing network complexity. A similar method was employed by Gali et al. [13] as well. Wang et al. [14] propose a 3D Enhanced Multi-scale Network (EMSN) that refines final prediction through a progressive auto-context procedure. They apply 3D dilated convolution, which enlarges the receptive field of the convolution kernel without loss of resolution. Zhang et al. [15] present a novel multitask framework that uses two segmentation networks. First, a coarse segmentation network with residual blocks localizes the regions of interest (ROI). After that, multi-level ROIs are cropped from the encoder part to form input to a decoder that provides fine, detail-preserving segmentation results. Kondratenko et al. [16] use 2D T-Net architecture and strong postprocessing that includes use of the non-intersecting packages division of each image. Feng et al. [17] use a simplified Dense V-Net. Similarly, Han et al. [18] proposed a multi-resolution VB-Net framework that reduces the computation cost while maintaining high segmentation accuracy. Moreover, a two-stage cascaded network presented by Kim et al. [19], uses the first network to select slices and the second network to provide final segmentations.

However, prior works in this area have focused only on improving the segmentation accuracy of organ structures. To our knowledge, none have considered the automatic construction of models suitable for CFD simulations. Thus, a need remains to develop an automated method to generate patient-specific CFD models and blood flow simulations directly from image data.

\subsection{Prior Aorta Simulation Methods}

Recently, numerous researches introduce numerical simulation methods to investigate blood flow structure, and its interaction with blood fluid [20]. These methods include the level set method [21], the fictitious domain method [22, 23], the immersed boundary method [24], the Fully Eulerian formulation [25] and the Arbitrary Lagrangian-Eulerian (ALE) [26, 27].

A plethora of methods for the simulation of blood pressure gradient of aortic coarctation models at rest and under exercise conditions were presented during the first and second Medical Image Computing and Computer-Assisted Intervention (MICCAI) CFD Challenge [28, 29]. Wang et al. [30] present haemodynamic metrics found in aortic dissection and quantitative indexes for optimal treatment planning. Moreover, Garje et al. [31] observed numerical changes in vessels' blood flow and their growth due to an accumulation of cholesterol in coronary artery disease (CAD). Methods for observing geometry and blood flow through an abdominal aortic aneurism allow timely prediction of aneurism ruptures and are described by Canchi [32]. Gonzales et al. [33] analyze the fluid dynamics that cause the wall stress distribution in abdominal aortic aneurysms, using accurate 3D geometry and a realistic, nonlinear, elastic biomechanical model. Lee et al.[34] introduce non-linear kernel support vector regression for prediction of AAA growth that requires only flow-mediated dilatation and AAA diameter as inputs. Selmi et al. [35] investigate blood flow in the upper aorta using Navier-Stokes equations and neo-Hookean hyperelastic model for describing vessels' wall behavior. Moreover, Caballero et al. [36] provides a comprehensive description of methods for anatomic and physiologic model creation and explains assumptions of assigning boundary conditions for simulations in the thoracic aorta. Fuchs et al. [37] focuses on the sensitivity of simulation results due to in- and outflow boundary conditions (BCs). Jia et al. [38] observes implications of using two different outlet boundary conditions in blood flow modelling based on using pressure or flow rate outlet boundary conditions. 


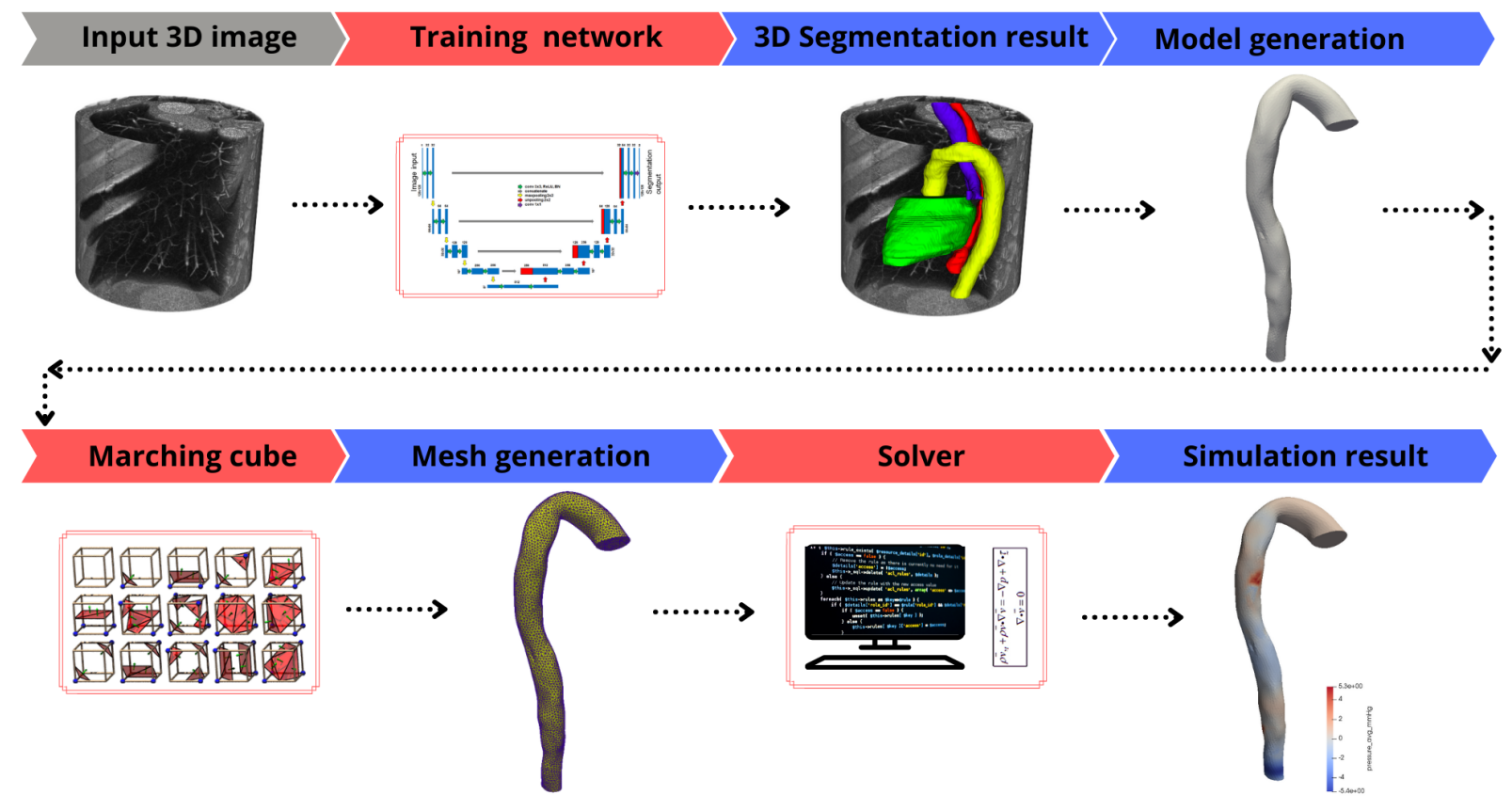

Figure 1. An illustration of a proposed four-stage framework for the simulation of blood flow in the thoracic aorta includes: (1) segmentation of 3D images, (2) model generation, (3) mesh generation, and (4) solving incompressible Navier-Stokes equations to simulate the blood flow in the aorta.

\section{Method}

The proposed automated framework consists of four major steps to generate CFD-compatible models for aorta flow simulations: (1) automatic segmentation, (2) model generation, (3) mesh creation, and (4) blood flow simulation. The whole workflow of our proposed framework is illustrated in Figure 1.

\subsection{Automated Segmentation}

Following the architecture of an original 3D U-Net, the 3D MultiResUnet structure has encoder and decoder paths. In the encoder path, there are three MultiRes blocks. Each MultiRes block has $3 \times 3 \times 3$ convolution layer with a $1 \times 1 \times 1$ residual connection, which is followed by strided convolution that increase feature size by 2 , and downsizes the image dimension by 2. A residual connection is added to gather more spatial information. Additionally, each MultiRes block contains the ReLU activation function and the Group Normalization (GN). Similarly, the decoder path contains MultiRes blocks, followed by strided convolution that decreases the feature size by 2 and upsizes the image dimension by 2 . Instead of commonly used skip connection for connecting encoder and decoder, ResPaths [39] are used. The strength of ResPath [39] is in a chain of $3 \times 3 \times 3$ filters with
$1 \times 1 \times 1$ residual connection that passes the feature maps from the encoder to the decoder, simultaneously concatenating them. The structure of an overall network, MultiRes blocks, and ResPath is shown in Figure 2.

\subsubsection{Training Details and Optimization}

We normalize each input 3D image based on the standard and mean deviation of their intensity values. To provide fine ROI for network input, we crop input images to a fixed size of $176 \times 224 \times 144$. For data augmentation, we apply random axis mirror flip with a probability of 0.5 , random scale, and intensity shift on the input image channel. We use $L 2$ norm regularization with a weight of $10^{-5}$, and employ the spatial dropout with a rate of 0.2 after the initial encoder convolution. We use Adam optimizer with an initial learning rate of $\alpha_{0}=10^{-4}$, and gradually decrease it according to the following expression:

$$
\alpha=\alpha_{0} *\left(1-\frac{c}{T_{c}}\right)^{0.9}
$$

where $T_{c}$ is a number of epochs and $c$ is an epoch counter. We employ a smoothed negative dice score loss function, which can be expressed as: 
Input image

Output

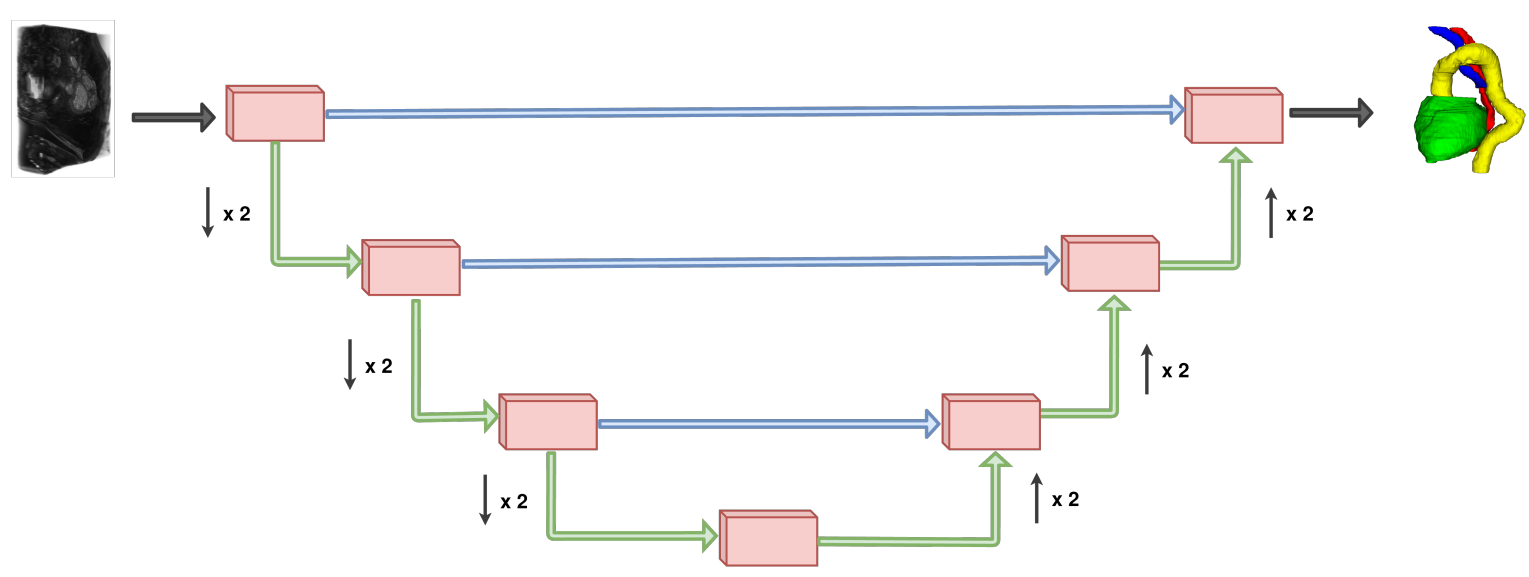

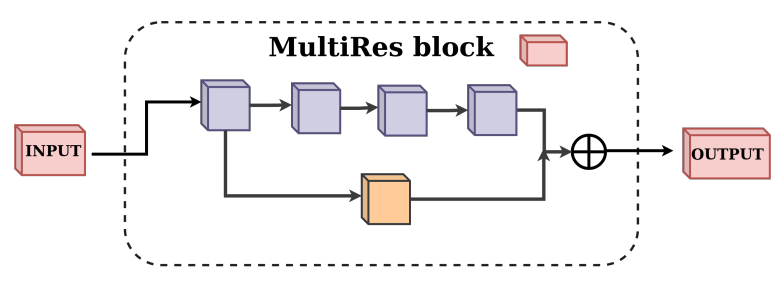

Convolution layer $3 \times 3 \times 3$

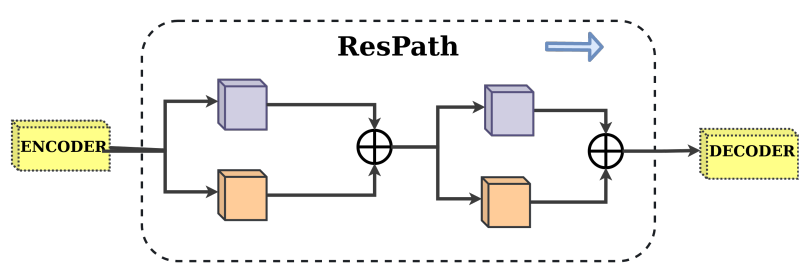

Convolution layer $1 \times 1 \times 1$ $\bigoplus$ Addition

Figure 2. An illustration of the architecture of 3D MultiResUNet, MultiRes block, and ResPath. MultiRes block consist of four $3 \times 3 \times 3$ convolution layers and a $1 \times 1 \times 1$ convolutional residual connection. ResPath has two $3 \times 3 \times 3$ convolutional layers each with one $1 \times 1 \times 1$ residual connection. The output layer uses the sigmoid activation function to obtain the final segmentation results for the trachea, esophagus, heart, and aorta.

$$
D_{\text {loss }}=-\frac{2 \sum_{i=1}^{N} p_{i} g_{i}+1}{\sum_{i=1}^{N} p_{i}+\sum_{i=1}^{N} g_{i}+1}
$$

where $p_{i}$ is probability of predicted regions, $g_{i}$ is the ground truth.

We use $80 \%-20 \%$ training and validation split, i.e., 32 volumetric images are used for training, and the 8 are used for validation purposes. A detailed description of the used dataset is given in Section 4.1. Final segmentation accuracy testing was done on an online CodaLab SegThor Challenge submission page on 20 patient subjects [40]. The total training time took approximately 12 hours on two NVIDIA Titan V GPUs simultaneously. The network is trained for 200 epochs since further training did not decrease validation loss as shown in Figure 3.

\subsection{Model generation and meshing}

We extract ascending and descending aorta regions from previously obtained segmentations and use
OpenCASCADE modeling kernel from SimVascular [41] to generate aorta models. After that, a marching cube algorithm was applied to generate a watertight surface mesh of the aorta. The obtained aorta was then triangulated using a constrained 2D Delaunay algorithm. First, the trimmed model is re-meshed with a maximum mesh edge size of $1.4 \mathrm{~mm}$. Second, a volume mesh is generated with an edge size of 1.8 $\mathrm{mm}$ and a boundary layer meshing near walls. With this procedure, we construct unstructured meshes of volumetric input segmentation. As such, they can be used as the computational domain to simulate the velocity and pressure of blood flow. An illustration of ascending and descending aorta with defined inlet and outlet boundaries and an example of obtained model and meshes is shown in Figure 4.

\subsection{Image-Based aorta CFD Simulations}

We apply the Arbitrary Lagrangian-Eulerian (ALE) formulation of the incompressible Navier-Stokes equations to simulate the blood flow. The continuity 


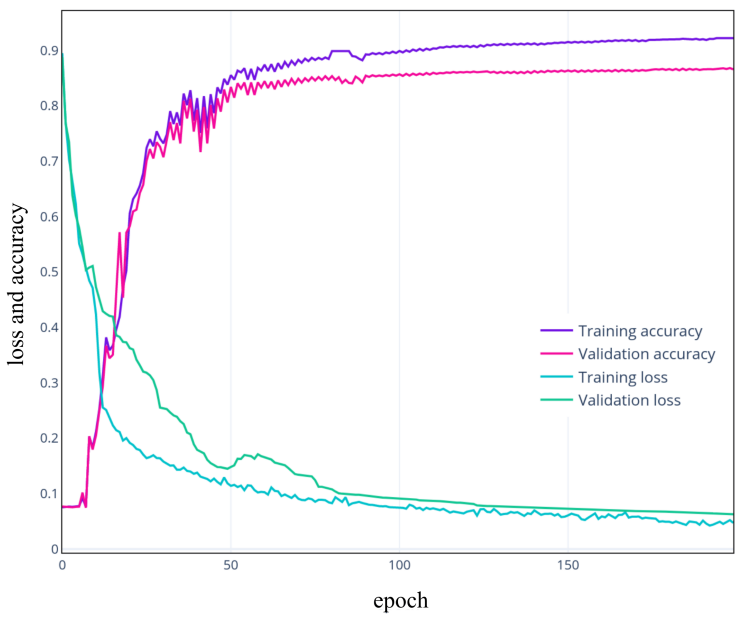

Figure 3. Training and validation accuracies and losses for 3D MultiResUnet network architecture. The network is trained for 200 epochs since further training did not decrease validation loss.

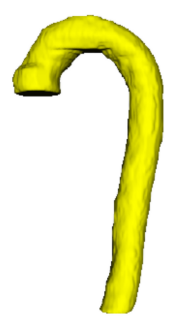

(a)

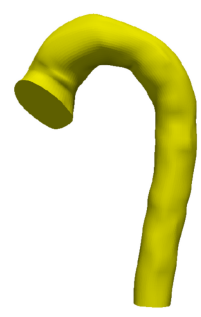

(b)

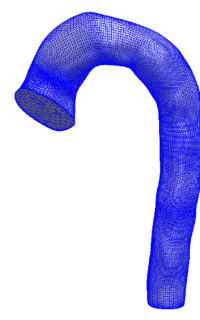

(c)
Figure 4. An example of obtained results of our proposed framework for: (a) aorta segmentation, (b) generated aorta model, and (c) created aorta mesh.

and momentum of Navier-Stokes equations written in the strong form can be expressed as follows:

$$
\begin{gathered}
v_{i, i}=0 \\
\dot{v}_{i}+v_{i, j}=-p_{, i}+\gamma_{i j, j}+f_{i}
\end{gathered}
$$

where $v_{i}$ is the $i-t h$ component of velocity, $p$ is the pressure that is assumed to be constant and is calculated with dividing by the density $\rho, f_{i}$ is the prescribed body force (divided by $\rho$ ), and $\gamma i j$ is the viscous stress tensor given with:

$$
\mu_{i j}=\gamma\left(v_{i, j}+v_{j, i}\right)
$$

where $\gamma=\frac{\mu}{\rho}$ is the kinematic viscosity. An arbitrary domain Navier-Stokes equations are solved using two methods: pressure-stabilizing Petrov Galerkin (PSPG) [42], and streamline-upwind Petrov-Galerkin
(SUPG) [43]. Specifically, this formulation is defined on the finite-dimensional weight function spaces and trial solution.

Let $\Phi \in R^{3}$ denote finate-dimensional domain with boundaries defined as $\Phi=\Phi D \cup \Phi N$ and weight function spaces $M_{h}^{k}, T_{h}^{k}$ and $O_{h}^{k}$. Neumann boundary conditions are applied on $\Phi N$, while Dirichlet boundary conditions are applied on $\Phi D$. By discretizing $\Phi$ with linear elements $\Phi_{e}$, Eq. 3 and Eq. 4 can be written as:

$$
\begin{array}{r}
B_{G}\left(t_{i}, o ; v_{i}, p\right)=\int_{\Phi}\left[t_{i}\left(\dot{v}_{i}+v_{j} v_{i, j}-f_{i}\right)\right. \\
\left.+t_{i, j}\left(-p \delta_{i j}+\gamma_{i j}\right)-o, i v_{i}\right] \Phi \\
+\int_{\Phi_{N}}\left[t_{i}\left(p \delta_{i n}-\gamma_{i n}\right)+q u_{n}\right] \Phi_{N}
\end{array}
$$

for all $t \in T_{h}^{k}$ and $o \in O$. The above equations were solved with the open-source svFSI solver from the SimVascular.

At the inlet boundary conditions, a time-varying flow rate was imposed, while at the outlet boundary conditions, a pressure wave was set. This information was adapted from [44]). The boundary conditions are defined in Figure 5. The blood was assumed to be Newtonian. The blood was assumed to be Newtonian. Blood was assumed to have a viscosity $\gamma=4.0$. $10^{-3}$ Pas and a density $\rho=1.06 \frac{\mathrm{g}}{\mathrm{cm}^{3}}$. Moreover, the vessel walls were assumed to be rigid for simplicity, and the no-slip condition was considered.

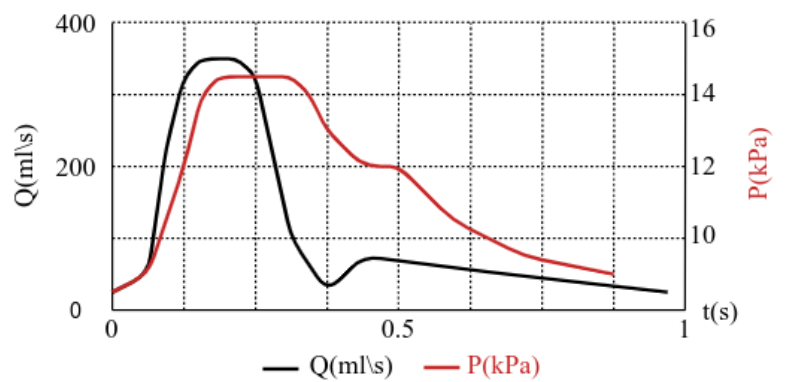

Figure 5. Graph showing inlet and outlet boundary conditions. Here, $Q$ is a flow rate, and $P$ is a preassure wave.

\section{Dataset and Results}

In this subsection, we describe the used dataset and provide obtained results for the segmentation and simulation parts of the proposed framework. 


\subsection{The SegTHOR Dataset}

In this work, we use a dataset provided by CodaLab SegTHOR Challenge [40]. The dataset is divided into train and test sets that include $40 \mathrm{CT}$ and $20 \mathrm{CT}$ volumetric images, respectively. This represents 7390 slices for training data and 3694 slices for test data. The number of slices differs between patients, and image sizes are between $512 \times 512 \times(150-284)$ voxels. The in-plane resolution varies between $0.90 \mathrm{~mm}$ and $1.37 \mathrm{~mm}$ per pixel, while the most common resolution is $0.98 \times 0.98 \times 2.5$. Each CT image in the train set has corresponding ground truth produced manually by an experienced radiotherapist. Ground truths of the test set are hidden, and evaluation is available through an online evaluation platform that calculates the Dice scores and the Hausdorff distance for each organ structure[40]. An example of input dataset image slices with corresponding ground truth is shown in Figure 6.

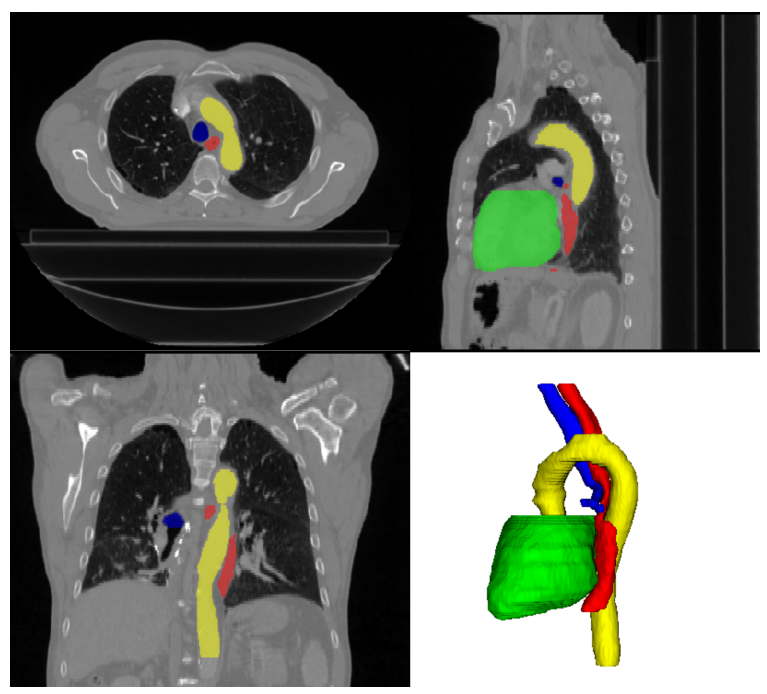

Figure 6. An example of an input image from the SegThor dataset. Top row: an axial slice of an original image with ground truth overlay (left) and a sagittal slice of an original image with ground truth overlay (right). Bottom row: a coronal slice of an original image with ground truth overlay (left) and 3D visualization (right).

\subsection{Segmentation and Simulation Results}

Segmentation accuracy was tested on the 40 patient CT scans from the CodaLab SegThor dataset with online evaluation framework [40] which computes Dice score (DSC) and Hausdorff distance (HD) between segmentation results and the (hidden) ground truth. The DSC metric measures the degree of overlap between the ground truth and predicted segmentation and is defined as follows:

$$
D S C(G, P)=\frac{2|G \cap P|}{|G|+|P|}
$$

On the other hand, HD represents the maximum of the minimum voxel-wise distances between the ground truth and predicted object boundaries and can be written as:

$$
H D(G, P)=\max _{g \in G}\left\{\min _{p \in P}\left\{\sqrt{g^{2}-p^{2}}\right\}\right\}
$$

where $g$ is the ground truth, and $p$ is the predicted mask.

Table 1 gives a comparison of the Dice score and Hausdorff distance of the esophagus, trachea, heart, and aorta produced by our automated segmentation framework with the results of other participants in the SegThor challenge. To provide further details on segmentation accuracy, the box plots in Figures 7 and Figure 8 give the distributions of the segmentation accuracy measures for all four structures.

We achieve an average Dice score of $69.84 \%$, $94.55 \%, 94.97 \%$, and $95.83 \%$ for esophagus, heart, trachea, and aorta, respectively. Our segmentation method performs exceptionally well for segmenting the trachea and aorta. In this paper, it is essential to achieve the highest possible accuracy for aortic segmentation since we need it in the following steps to generate models and simulations. Therefore, the obtained dice score of $95.83 \%$ and low HD of 0.3021 support our design choice of using 3D MultiResUnet network architecture for aortic segmentation. Moreover, heart results are comparable to the other challenge algorithms. However, the use of a very deep network with a large number of feature maps appears not suitable for the segmentation of the esophagus.

While testing the accuracy of the segmentation process is important, it only assesses the accuracy of pixel classification. This does not directly guarantee that segmentations will lead to valid geometries from which effective meshes could be obtained. To ensure our geometry reconstruction and mesh generation process's robustness, we visually inspect all 20 obtained CT segmentations from the SegTHOR test set. After that, we check whether there were any errors in geometry construction or volumetric meshing and visually inspected the models for apparent artifacts. For all obtained segmentation results from $20 \mathrm{CT}$ patient scans, our framework generated the reconstructed aorta geometry and produced a valid volumetric mesh.

Figure 9 shows pressure, displacement, and velocity streamlines in the ascending and descending aorta. The lowest pressure is in an ascending aorta, and it 
Table 1. Comparison of dice scores and Hausdorff distances of state-of-the-art methods and our proposed method for esophagus, heart, trachea and aorta segmentation.

\begin{tabular}{|c|c|c|c|c|c|c|c|c|c|}
\hline Authors & Method & Esophagus & $\begin{array}{l}\text { DSC } \\
\text { Heart }\end{array}$ & Trachea & Aorta & Esophagus & $\begin{array}{l}\text { HD } \\
\text { Heart }\end{array}$ & Trachea & Aorta \\
\hline Chen et al. [10] & 2DU-Net & 0.8166 & 0.9329 & 0.8910 & 0.9232 & 0.4914 & 0.2417 & 0.2746 & 0.3081 \\
\hline He et al. [11] & 2D Residual U-Net & 0.8594 & 0.9500 & 0.9201 & 0.9484 & 0.2743 & 0.1383 & 0.1824 & 0.1129 \\
\hline Gali et al. [13] & Dilated U-Net & 0.4648 & 0.8597 & 0.6295 & 0.8526 & 2.8665 & 0.8993 & 3.9841 & 1.4577 \\
\hline Wang et al. [14] & EMSN & 0.8386 & 0.9310 & 0.9067 & 0.9352 & 0.3637 & 0.2874 & 0.2670 & 0.2517 \\
\hline Zhang et al. [15] & Loc + seg & 0.7732 & 0.9384 & 0.8939 & 0.9232 & 1.6774 & 0.2089 & 0.2741 & 0.3081 \\
\hline Kondratenko et al. [16] & 2D T-Net & 0.80 & 0.93 & 0.89 & 0.92 & 0.62 & 0.30 & 0.81 & 0.27 \\
\hline Feng et al. [17] & Dense V-Net & 0.7734 & 0.9414 & 0.8927 & 0.9233 & 0.6400 & 0.1821 & 0.3077 & 0.2357 \\
\hline Kim et al. [19] & Cascaded Network & 0.7518 & 0.9328 & 0.8885 & 0.8919 & 0.9267 & 0.2184 & 0.6164 & 1.1300 \\
\hline Proposed & 3D MultiResUnet & 0.6984 & 0.9455 & 0.9497 & 0.9583 & 0.9435 & 0.2217 & 0.3357 & 0.3021 \\
\hline
\end{tabular}

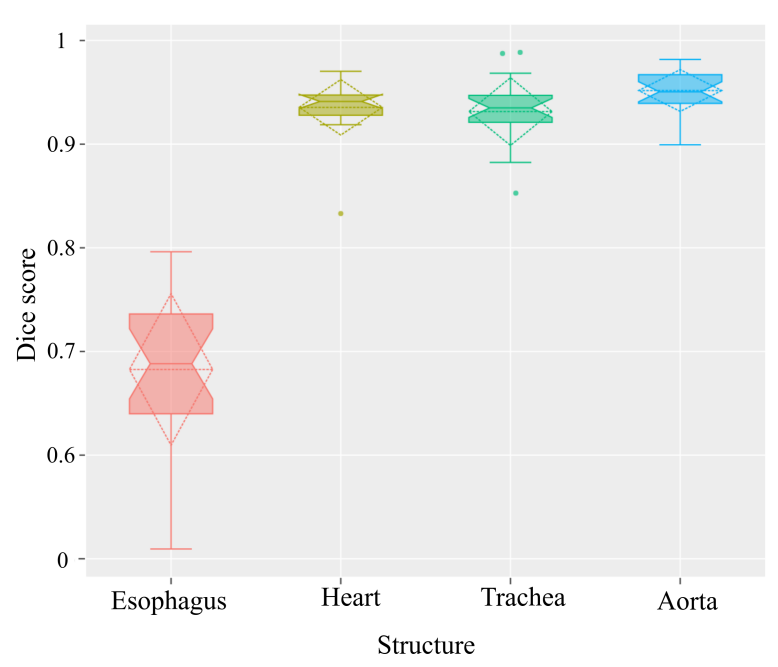

Figure 7. Box plots of dice scores for esophagus, heart, trachea and aorta of segmentation results from the SegThor test data sets.

slowly increases in the aortic arch, specifically on the upper aortic arch. Conversely, the maximum pressure distribution is seen in descending aorta. Similarly, the velocity is lower in the ascending aorta while the peak velocity blood flow is obtained in descending aorta, i.e., at the outlet. The obtained results are in the expected range for ascending and descending aorta, thus guarantee the correctness of the proposed approach.

\section{Conclusion}

In this work, we have presented a framework for fully automatic CFD simulation through the aorta from patient-specific CT images. The framework combines novel deep-learning-based automatic segmentation algorithms and geometry processing algorithms to create simulation-ready CFD-suitable models from CT image data and explore blood flow simulations. Specifically, it contains four major stages: (1) automatic segmentation of the aorta, (2) model generation, (3) mesh creation, and (4)

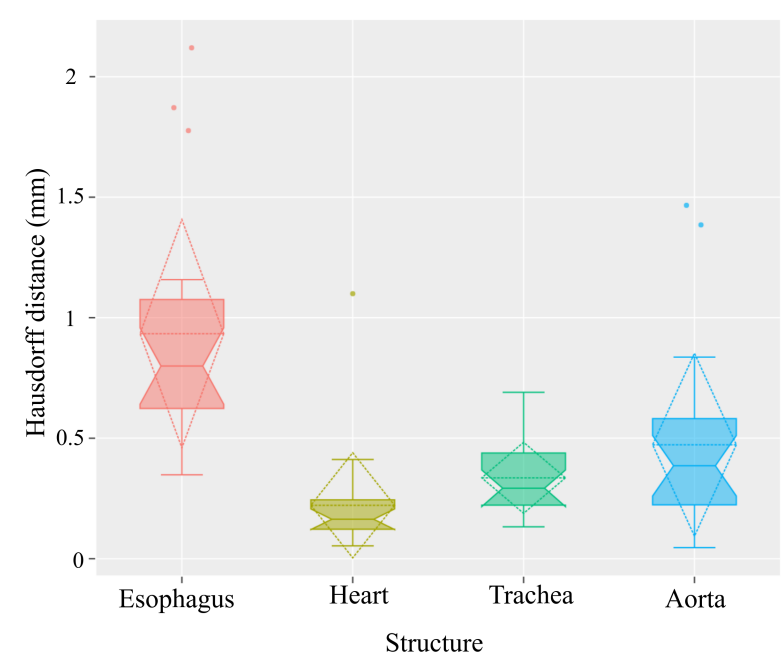

Figure 8. Box plots of Hausdorff distance for esophagus, heart, trachea and aorta of segmentation results from the SegThor test data sets.

blood flow simulation. In the segmentation part, we utilized a 3D MultiResUnet network for automatic segmentation of organs at risk from the CodaLab SegThor Challenge. Segmentation results are highly comparable to the current state-of-the-art, yielding DSC for esophagus, heart, trachea, and aorta of $69.84 \%$, $94.55 \%, 94.97 \%$, and $95.83 \%$, respectively. After successful segmentation, ascending and descending aorta are extracted and processed to obtain aortic models and meshes. To support CFD simulation of aorta hemodynamics using an ALE formulation, the framework can automatically identify boundary faces of mitral and aortic opening. We simulate the pressure along the surface of the aorta, displacement, and velocity. Our framework offers orders of magnitude savings in time and human efforts in developing image-based CFD simulations of aortic flow compared with prior manual or semi-automatic methods. The entire framework was implemented in Python with open-sourced dependencies (Pytorch, VTK, 


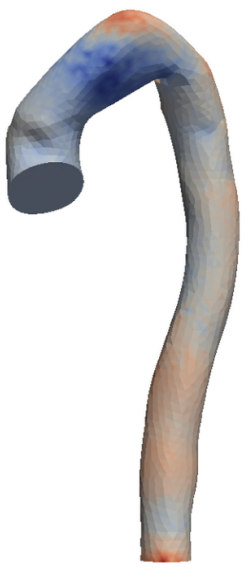

(a)
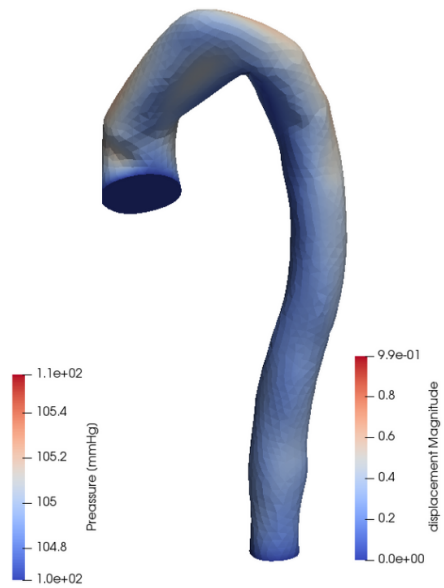

(b)

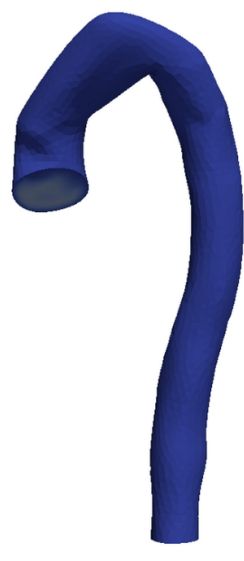

(c)

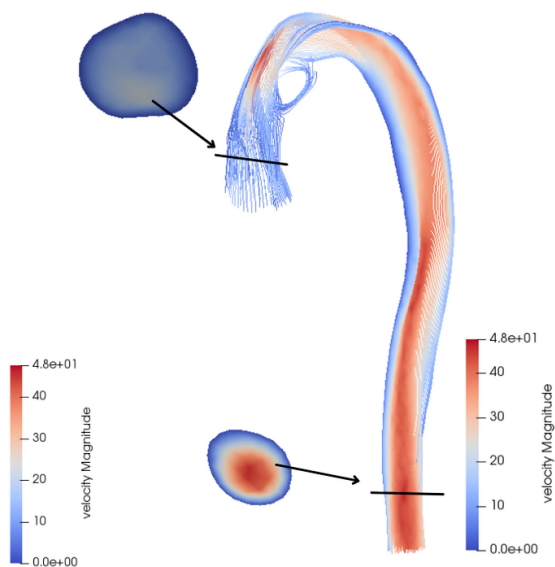

(d)

Figure 9. Simulation results for: (a) preassure, (b) displacement, (c) velocity, (d) velocity stream with examples of velocity slices at inlet and outlet.

SimVascular, SimpleITK), can be executed from the command line, and does not require user intervention, significantly reducing aorta CFD simulation time.

\section{Acknowledgments}

This work has been supported in part by Croatian Science Foundation under the project UIP-2017-05-4968.

\section{References}

[1] W. H. Organization, "Mortality database," 2018. [Online; accessed 19-January-2021].

[2] C. Warboys, N. Amini, A. Luca, and P. Evans, "The role of blood flow in determining the sites of atherosclerotic plaques," F1000 medicine reports, vol. 3, p. 5, 032011.

[3] D. Pleouras, A. Sakellarios, P. Tsompou, V. Kigka, S. Kyriakidis, S. Rocchiccioli, D. Neglia, J. Knuuti, G. Pelosi, L. Michalis, and D. Fotiadis, "Simulation of atherosclerotic plaque growth using computational biomechanics and patient-specific data," Scientific Reports, vol. 10, 102020.

[4] L. Zhong, J.-M. Zhang, B. Su, R. S. Tan, J. C. Allen, and G. S. Kassab, "Application of patient-specific computational fluid dynamics in coronary and intra-cardiac flow simulations: Challenges and opportunities," Frontiers in Physiology, vol. 9, p. 742, 2018.

[5] P. Jankowski and D. Czarnecka, "Pulse pressure, blood flow, and atherosclerosis," American Journal of Hypertension, vol. 25, pp. 1040-1041, 102012.

[6] B. Šeta, M. Torlak, and A. Vila, "Numerical simulation of blood flow through the aortic arch," in $C M B E B I H$ 2017 (A. Badnjevic, ed.), (Singapore), pp. 259-268, Springer Singapore, 2017.
[7] S. Numata, K. Itatani, K. Kanda, K. Doi, S. Yamazaki, K. Morimoto, K. Manabe, K. Ikemoto, and H. Yaku, "Blood flow analysis of the aortic arch using computational fluid dynamics $\dagger$, , European Journal of Cardio-Thoracic Surgery, vol. 49, pp. 1578-1585, 01 2016.

[8] O. Ronneberger, P. Fischer, and T. Brox, "U-net: Convolutional networks for biomedical image segmentation," CoRR, vol. abs/1505.04597, 2015.

[9] Ö. Çiçek, A. Abdulkadir, S. S. Lienkamp, T. Brox, and O. Ronneberger, "3d u-net: Learning dense volumetric segmentation from sparse annotation," CoRR, vol. abs/1606.06650, 2016.

[10] P. Chen, C. Xu, X. Li, Y. Ma, and F. Sun, "Two-stage network for oar segmentation," in SegTHOR@ISBI, 2019.

[11] T. He, J. Guo, J. Wang, X. Xu, and Z. Yi, "Multi-task learning for the segmentation of thoracic organs at risk in ct images," in SegTHOR@ISBI, 2019.

[12] S. Vesal, N. Ravikumar, and A. Maier, "A 2d dilated residual u-net for multi-organ segmentation in thoracic ct," ArXiv, vol. abs/1905.07710, 2019.

[13] M. S. K. Gali, N. Garg, and S. Vasamsetti, "Dilated u-net based segmentation of organs at risk in thoracic ct images,"in SegTHOR@ISBI, 2019.

[14] Q. Wang, W. Zhao, C. Zhang, L. Zhang, C. Wang, Z. Li, S. Cui, and G. Li, "3d enhanced multi-scale network for thoracic organs segmentation," in Proceedings of the 2019 Challenge on Segmentation of THoracic Organs at Risk in CT Images, SegTHOR@ISBI 2019, April 8, 2019 (C. Petitjean, S. Ruan, Z. Lambert, and B. Dubray, eds.), vol. 2349 of CEUR Workshop Proceedings, CEUR-WS.org, 2019.

[15] L. Zhang, L. Wang, Y. Huang, and H. Chen, "Segmentation of thoracic organs at risk in ct images combining coarse and fine network," in SegTHOR@ISBI, 2019. 
[16] V. Kondratenko, D. Denisenko, A. Pimkin, and M. Belyaev, "Segmentation of thoracic organs at risk in ct images using localization and organ-specific cnn," CEUR Workshop Proceedings, vol. 2349, 2019.

[17] M. Feng, W. Huang, Y. Wang, and Y. Xie, "Multi-organ segmentation using simplified dense v-net with post-processing," in SegTHOR@ISBI, 2019.

[18] M. Han, G. Yao, W. Zhang, G. Mu, Y. Zhan, X. Zhou, and Y. Gao, "Segmentation of ct thoracic organs by multi-resolution vb-nets," in SegTHOR@ISBI, 2019.

[19] S. Kim, Y. Jang, K. Han, H. Shim, and H.-J. Chang, "A cascaded two-step approach for segmentation of thoracic organs," inSegTHOR@ISBI, 2019.

[20] Z. Li and J. Gillard, "Simulation of the interaction between blood flow and atherosclerotic plaque," 2007 29th Annual International Conference of the IEEE Engineering in Medicine and Biology Society, pp. 1699-1702, 2007.

[21] Y. Chang, T. Hou, B. Merriman, and S. Osher, "A level set formulation of eulerian interface capturing methods for incompressible fluid flows," Journal of Computational Physics, vol. 124, no. 2, pp. 449-464, 1996.

[22] R. Glowinski, T.-W. Pan, and J. Periaux, "A fictitious domain method for dirichlet problem and applications," Computer Methods in Applied Mechanics and Engineering, vol. 111, no. 3, pp. 283-303, 1994.

[23] R. Glowinski, T.-W. Pan, and J. Periaux, "A fictitious domain method for external incompressible viscous flow modeled by navier-stokes equations," Computer Methods in Applied Mechanics and Engineering, vol. 112, no. 1, pp. 133-148, 1994.

[24] C. S. Peskin, "Numerical analysis of blood flow in the heart," Journal of Computational Physics, vol. 25, no. 3, pp. 220-252, 1977.

[25] T. Wick, "Flapping and contact fsi computations with the fluid-solid interface-tracking/interface-capturing technique and mesh adaptivity," Computational Mechanics, vol. 53, 102013.

[26] T. J. Hughes, W. K. Liu, and T. K. Zimmermann, "Lagrangian-eulerian finite element formulation for incompressible viscous flows," Computer Methods in Applied Mechanics and Engineering, vol. 29, no. 3, pp. 329-349, 1981.

[27] J. Donea, S. Giuliani, and J. Halleux, "An arbitrary lagrangian-eulerian finite element method for transient dynamic fluid-structure interactions," Computer Methods in Applied Mechanics and Engineering, vol. 33, no. 1, pp. 689-723, 1982.

[28] Medical Image Computing and Computer-Assisted Intervention - MICCAI 2012 15th International Conference, Nice, France, October 1-5, 2012, Proceedings, Part II. Springer Publishing Company, Incorporated, 2012.

[29] O. Camara, T. Mansi, and M. Pop, Statistical Atlases and Computational Models of the Heart - Imaging and Modelling Challenges: 5th International Workshop, STACOM 2014, Held in ... September 18, 2014, Revised Selected Papers. Springer Publishing Company, Incorporated, 2015.

[30] Q. Wang, X. Guo, E. Poon, A. Ooi, and R. P., "Three-dimensional cfd simulation of blood flow in aortic dissection," 122020.
[31] A. Garje, Y. G. Adhav, and D. Bodas, "Design and simulation of blocked blood vessel for early detection of heart diseases," in 2015 2nd International Symposium on Physics and Technology of Sensors (ISPTS), pp. 204-208, 2015.

[32] T. Canchi, D. K. Srinivasan, E. Ng, and S. Narayanan, "A review of computational methods to predict the risk of rupture of abdominal aortic aneurysms," BioMed Research International, vol. 2015, 102015.

[33] M. Gonzalez-Urquijo, R. Zamacona, A. Karen, M. Mendoza, M. Iribarren, E. Ibarra, M. David, M. Moya Bencomo, and M. A. Fabiani, "3d modeling of blood flow in simulated abdominal aortic aneurysm," Vascular and Endovascular Surgery, 042021.

[34] R. Lee, D. Jarchi, R. Perera, A. Jones, I. Cassimjee, A. Handa, D. Clifton, K. Bellamkonda, F. Woodgate, N. Killough, N. Maistry, A. Chandrashekar, C. Darby, A. Halliday, L. Hands, P. Lintott, T. Magee, A. Northeast, J. Perkins, and E. Sideso, "Applied machine learning for the prediction of growth of abdominal aortic aneurysm in humans," EJVES Short Reports, vol. 39, pp. 24-28, 2018.

[35] M. Selmi, H. Belmabrouk, and A. Bajahzar, "Numerical study of the blood flow in a deformable human aorta," Applied Sciences, vol. 9, no. 6, 2019.

[36] A. Caballero and S. Lain, "A review on computational fluid dynamics modelling in human thoracic aorta," Cardiovascular Engineering and Technology, vol. 4, 06 2013.

[37] A. Fuchs, N. Berg, and L. Prahl Wittberg, "Pulsatile aortic blood flow-a critical assessment of boundary conditions," Journal of Engineering and Science in Medical Diagnostics and Therapy, vol. 4, 112020.

[38] Y. Jia, K. Punithakumar, M. Noga, and A. Hemmati, "The implications of two outlet boundary conditions on blood flow simulations in normal aorta of pediatric subjects," Theoretical and Computational Fluid Dynamics, vol. 35, 062021.

[39] N. Ibtehaz and M. S. Rahman, "Multiresunet : Rethinking the u-net architecture for multimodal biomedical image segmentation," CoRR, vol. abs/1902.04049, 2019.

[40] "Segthor codalab challenge." https: // competitions.codalab.org/ competitions/21145. Accessed: 2021-03-30.

[41] A. Updegrove, N. Wilson, J. Merkow, H. Lan, A. Marsden, and S. Shadden, "Simvascular: An open source pipeline for cardiovascular simulation," Annals of Biomedical Engineering, vol. 45, 122016.

[42] C. Whiting and K. Jansen, "A stabilized finite element method for the incompressible navier-stokes equations using a hierarchical basis," International Journal for Numerical Methods in Fluids, vol. 35, pp. 93-116, 2001.

[43] A. N. Brooks and T. J. Hughes, "Streamline upwind/petrov-galerkin formulations for convection dominated flows with particular emphasis on the incompressible navier-stokes equations," Computer Methods in Applied Mechanics and Engineering, vol. 32, no. 1, pp. 199-259, 1982.

[44] J. Alastruey, N. Xiao, H. Fok, T. Schaeffter, and C. Figueroa, "On the impact of modelling assumptions in multi-scale, subject-specific models of aortic haemodynamics," Journal of The Royal Society Interface, vol. 13, p. 20160073, 062016. 